\title{
Tumor-derived CD4+CD25+ Tregs Inhibit the Maturation and Antigen-Presenting Function of Dendritic Cells
}

\author{
Yong Du ${ }^{1}$, Xin Chen ${ }^{2}$, Xiu-Qing $\mathrm{Lin}^{2}$, Wei Wu${ }^{2}$, Zhi-Ming Huang ${ }^{2 *}$
}

\begin{abstract}
CD4+CD25+regulatory $T$ cells (Tregs) play a key role in regulation of immnue response and maintenance of self-tolerance. Studies have found Tregs could suppress tumor-specific $T$ cell-mediated immune response and promote cancer progression. Depletion of Tregs can enhance antitumor immunity. Dendritic cells (DCs) are professional antigen-presenting cells and capable of activating antigen-specific immune responses, which make them ideal candidate for cancer immunotherapy. Now various DC vaccines are considered as effective treatment for cancers. The aim of this study was to evaluate variation of Tregs in BALB/C mice with hepatocellular carcinoma and investigate the interaction between tumor-derived Tregs, effector $T$ cells (Teff) and splenic DCs. We found the percentages of Tregs/CD4+ in the peripheral blood of tumor-bearing mice were higher than in normal mice. Tumor-derived Tregs diminished the up-regulation of costimulatory molecule expression on splenic DCs, even in the presence of Teff cells and simultaneously inhibited IL-12 and TNF- $\alpha$ secretion by DCs.
\end{abstract}

Keywords: CD4+CD25+regulatory T cell - dendritic cell - costimulatory molecule - immunoregulation

Asian Pac J Cancer Prev, 16 (7), 2665-2669

\section{Introduction}

Hepatocellular carcinoma (HCC) is one of the most prevalent cancers worldwide and the second leading cause of cancer deaths for men while the sixth for women (Jemal et al., 2011) . Patients with cirrhosis are at highest risk of developing this malignant disease (Forner et al., 2012). Tregs have been confirmed to participate not only in autoimmunity, graft rejection but also in tumors. An increased percentage of Tregs in CD4+ T cells has been found in peripheral blood and tumour infiltrating lymphocytes of cancer patients, and high level of Tregs is an indicator of poor prognosis (Shen et al., 2010; Zhan et al., 2012; Hu et al., 2013; Wang et al., 2013). Tregs suppress antitumor immunity and are major obstacles for cancer immunotherapy.

Dendritic cell (DC) which is considered to represent the most potent antigen-presenting cell (APC) is the initiator and modulator of the immune response. Now various DC vaccines are considered as effective treatment for cancers (Hu et al., 2013; Ramanathan et al., 2014; Ahmed et al., 2014). A substantial number of researches proved that Tregs could preferentially form aggregates around DCs and inhibit the function of DCs through down-modulation of B7-molecules which required for activation and expansion of Teff cells and inhibition of proinflammatory cytokines secretion by DCs (Onishi et al., 2008; Herman et al., 2012). So the DCs could not elicit an effective antigen-specific T-cell response.
With in-depth research on Tregs, it was discoverd that there were some difference between tumor-derived Tregs and Tregs from normal controls. Tumor-derived Tregs are functionally distingguishable from naive Tregs (Liu et al., 2009). Tregs from HCC patients which might have a higher state of preactivation were more suppressive than Tregs from controls, and expressed more TGF $\beta 1$ (Unitt et al., 2005; Cabrera et al., 2010). Little is known about the interaction between Treg, Teff, and DC; and the change of Tregs in patients with hepatocellular carcinoma. To address these issues, we have evaluated the inhibitory potential of Tregs derived from HCC mice on splenic DCs and in this report, we have futher investigated the cellular and molecular mechnism between Treg, Teff, and DC. We demonstrated that there was an increase of Tregs/ CD4+ proportion in the peripheral blood of HCC mice. The inhitbition function of Tregs was not influenced by Teff cells and down-regulation of CD80/86 occured even in the presence of Teff cells.

\section{Materials and Methods}

\section{Mice}

Male BALB/C mice (6-8 weeks old, purchased from the SLACCAS company, China) were bred under specific pathogen-free (SPF) conditions. Animal protocols were approved by the Experimental Animal Management Committee Of Wenzhou Medical University. All surgery was performed under chloralic hydras anesthesia, and all 
efforts were made to minimize suffering.

\section{Reagents and materials}

Mouse CD4+CD25+regulatory T cell isolation kit and mouse CD11c magnetic bead sorting kit (MACS, Miltenyi Biotec, Germany); Fluorescentlylabeled antibody: CD4FITC, CD25-PE, CD11c-FITC, CD86-PE, CD80-PE and corresponding homotype antibody (eBioscience); Mouse TNF- $\alpha$, IL-12 ELISA kit (IBL, Germany); AntiCD3 antibody (BD biosciences); LPS (sigma); FCS and RPMI1640 (Gibco); mouse H22 hepatocellular carcinoma cell lines (China Center for Type Culture Collection, wuhan, $\mathrm{CN}$ ); Flow cytometry (BD).

\section{Tumor model and Histopathological examination}

The H22 hepatocellular carcinoma cells were cultured in RPMI 1640 medium.Then the cells were inoculated to abdomen with $1 \times 10^{6}$ cells per mouse. After 8-9 days, cancerous ascites were extracted aseptically. Cells were resuspended to a concentration of $1 \times 10^{6} / \mathrm{ml}$ for making model. The mice were randomly divided into the tumor group and control group. After the mouse was celiac anesthesia, we did a longitudinal incision, exposed the liver, injected $0.01 \mathrm{ml}$ cancer cell suspension $\left(10^{4}\right.$ cells $)$ to the liver and last closed enterocoelia. About 25 days later, the models were formed. When anatomy, we could see gray and nubbly carcinoma tissues at different sizes from 0.5 to $1.0 \mathrm{~cm}$ in diameters in livers. $4-\mu \mathrm{m}$ thick sections were prepared and stained with haematoxylin and eosin by standard histological procedures. Through the histopathological examination, we could confirm the model construction successful (Figure 1).

\section{Sample preparation and flow cytometry}

Mouse peripheral blood was collected from orbital plexus and anticoagulated with sodium heparin. The samples were stained with CD4-FITC and CD25-PE antibodies at $4^{\circ} \mathrm{C}$ for 30 minutes. Then erythrocytes were lysed by red blood cell lysis buffer. After washed with PBS, the samples were fixed and analyzed by flow cytometry. Isotype-matched antibodies were used as controls.

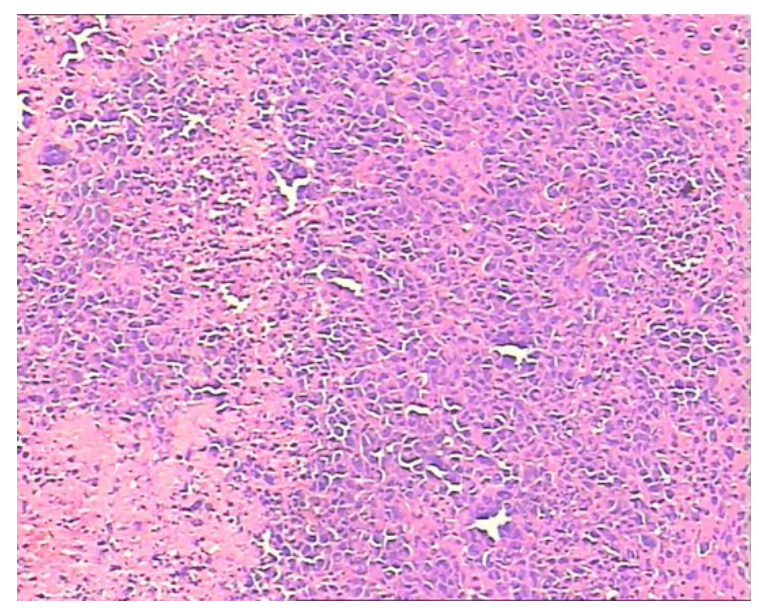

Figure 1. Histopathological Examination of Carcinoma

Tissue. The liver normal structure was lost and cells were pleomorphism obviously, arranging disorderly, necrosis visibly (magnification $\times 200$ )

\section{Cell isolation and sorting}

CD4+CD25+Tregs were enriched from HCC mice spleens. Splenic single-cell suspension was prepared from tumor-bearing mice and then tumor-derived Tregs were purified by magnetic cell sorting using mouse CD4+CD25+regulatory $\mathrm{T}$ cell isolation kit according to the manufacturers' instructions. First, through the negative sorting, CD4+T cells were got; then through positive sorting, CD4+CD25+T cells and CD4+CD25- Teff cells could be got respectively. Purity of sorted CD4+CD25+T cells or CD4+CD25- Teff cells were $>90 \%$ or $>95 \%$ respectively (Figure2A). Both $\mathrm{T}$ cells were stimulated separately with plate-bound anti-CD3 antibody $(1 \mu \mathrm{g} / \mathrm{ml})$ and IL-2 $(10 \mathrm{U} / \mathrm{ml})$ at $5 \times 10^{5} / \mathrm{ml}$ for $24 \mathrm{~h}$ in $24-w e l l$ plates. Splenic DCs were enriched from the spleen of normal mice using CD11c-conjugated microbeads according to the manufacturers' instructions. For highst recovery and purity, spleen single-cell suspensions had to be prepared by enzymatic disaggregation with Collagenase D. $>95 \%$ cells were positive for CD11c as assessed by flow cytometry (Figure2A).

\section{Cell cultures}

All cells were cultured in RPMI 1640 media supplemented with $10 \%$ fetal calf serum, $100 \mathrm{U} / \mathrm{ml}$ of penicillin $\mathrm{G}$ and $100 \mu \mathrm{g} / \mathrm{ml}$ of streptomycin in humidified $\mathrm{CO} 2$ incubator at $37^{\circ} \mathrm{C}$. Preactivated Tregs $\left(4-8 \times 10^{5} / \mathrm{ml}\right)$ or Teff cells $\left(4-8 \times 10^{5} / \mathrm{ml}\right)$ or a mix of two population at a $1: 1$ ratio were cultured with splenic DCs (DC-T cell ratio of $1: 2)$ for $24 \mathrm{~h}$ and LPS was added $(1 \mu \mathrm{g} / \mathrm{ml})$ to stimulate DCs for another $24 \mathrm{~h}$. Control DCs were in medium alone. After coculture, the splenic DCs were isolated, stained with relevant $\mathrm{mAbs}$ and analyzed by flow cytometry. The co-cultures and the single cell culture supernatants were collected. TNF- $\alpha$ and IL-12 were assessed by ELISA using corresponding enzyme-linked immunosorbent assay kits according to the manufacturer's instruction.

\section{Statistical analysis}

Data are expressed as mean \pm sd. Statistical analysis was

\section{A}
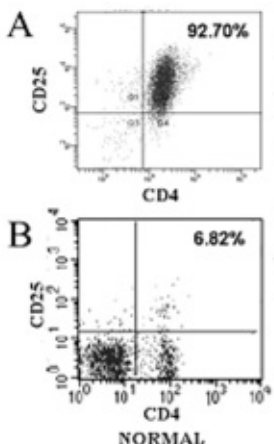

NORMAL
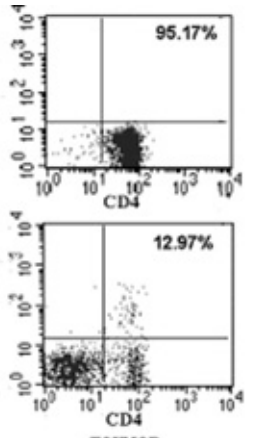

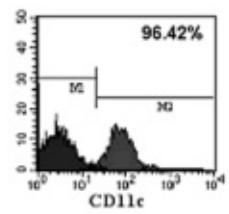

Figure 2. Representative Figures of Flow Cytometry

Examination. (A) CD4+T cells from HCC-mice spleen were sorted into CD25- and CD25+ cells by immunomagnetic beads. The purities of Tregs or Teff cells were $>90 \%$ or $>95 \%$ respectively. DCs from BALB/C mice spleen were isolated by CD11C+immunomagnetic beads and the purity was $>95 \%$. (B) The representative figures of protortions of $\mathrm{CD} 4+\mathrm{CD} 25+/ \mathrm{CD} 4+$ in peripheral blood from normal BALB/C mice or $\mathrm{HCC}$ mice. Peripheral blood lymphocytes were stained with anti-CD4 and anti-CD25 antibodies 
performed using Mann-Whitney U test and Student's t test with SPSS18.0 program (SPSS, Chicago). All P values $<0.05$ were considered statistically significant.

\section{Results}

Increase frequency of Tregs in the peripheral blood of HCC mice

To investigate the changes of the proportion of CD4+CD25+/ CD4+ in tumor bearing mice, $1 \times 10^{4} \mathrm{H} 22$ hepatocellular carcinoma cells were inoculated in the liver of BALB/C mice respectively $(n=10)$, while to evaluate the normal proportion, 10 normal mice were used as control group. Then the peripheral blood samples were collected and analyzed by flow cytometry using antimouse CD4 and CD25 antibodies. We found an increase in the proportion of CD4+CD25+/CD4+ in tumor bearing mice compared with that in normal mice $(10.05 \pm 1.86 \%$ vs $6.07 \pm 1.07 \%, p<0.05)$. The representable figures of the proportion from tumor-bearing mice or normal mice were shown in Figure 2B.

Tumor-derived Tregs can down-regulate costimulatory molecules expression on splenic DCs

The expression of costimulatory molecules are
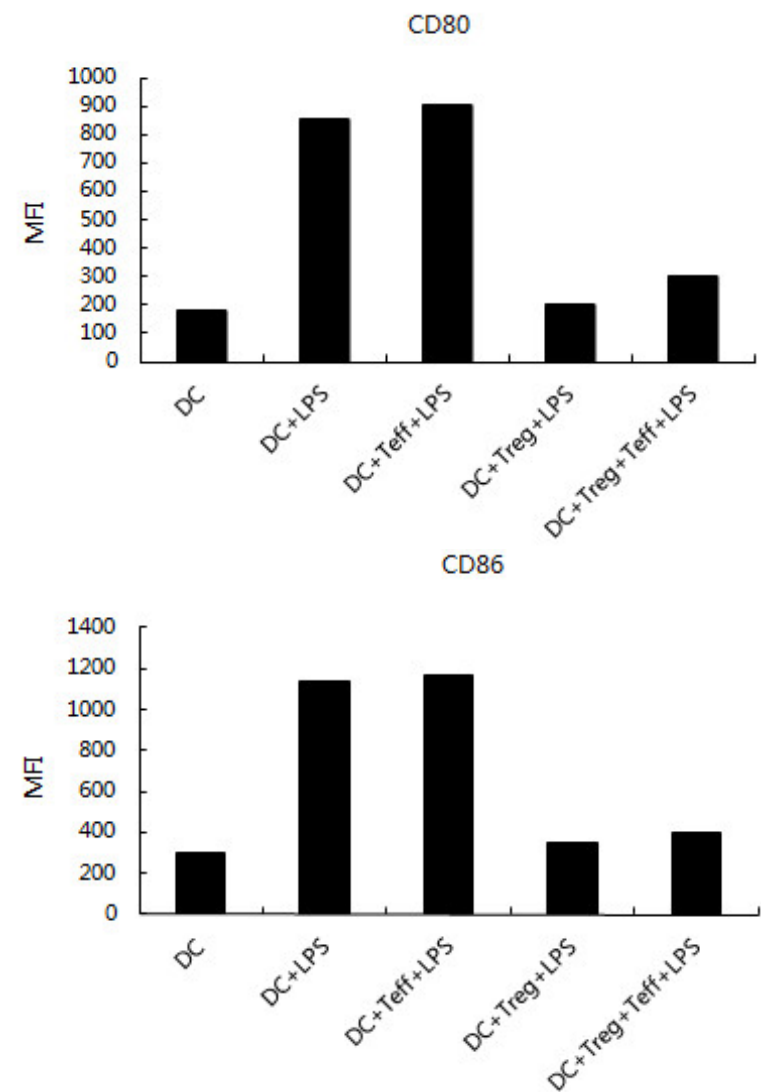

Figure 3. Tregs Down-Regulated CD80/86 Expression Even in the Presence of Teff Cells. Splenic DCs were cultured in medium, or with Tregs or Teff cells (DC-T cell ratio of 1:2 ) or a mix of both two cells at a 1:1 ratio, and then stimulated by LPS $(1 \mu \mathrm{g} / \mathrm{ml})$. Then DCs were isolated by fow cytometry and the CD80/86 expression on DCs was analyzed. Tumor-derived Tregs inhibit the up-regulation of CD80/86 expression on DCs even in the DC, Treg and Teff mixed culture group. The data show mean fluorescence intensity (MFI) of gated CD11c positive cells. Each graph represents three independent experiments associated with the maturation status of DCs. In our previous experiments, we have found tumor-derived Tregs could down-regulate CD80/86 expression on bone marrow-derived DCs in vitro, but we did not how HCCderived Tregs affect the function of splenic DCs in the presence of Teff cells. To study the interaction between DC, Treg and Teff, splenic DCs were co-cultured in medium alone, or with Tregs, or with Teff cells, or with a mix of Teff and Tregs at a 1:1 ratio. Then the expression of CD80/86 on DCs was examined by flow cytometry. Freshly isolated splenic DCs expressed low levels of costimulatory molecules CD80/86. When DCs were activated by LPS, obvious up-regulation of CD80/86 was observed. Teff cells could increase the expression of the costimulatory molecules on DCs. Compared with control DCs cultured in medium alone, splenic DCs co-cultured with Tregs showed a decline in CD80/86 expression while DCs co-cultured with Teff cells higher expressed CD80/86. Tumor-derived Tregs down-regulated costimulatory molecules expression on splenic DCs even in the presence of Teff cells (Figure 3). This may explain why effective anti-tumor immune can not be
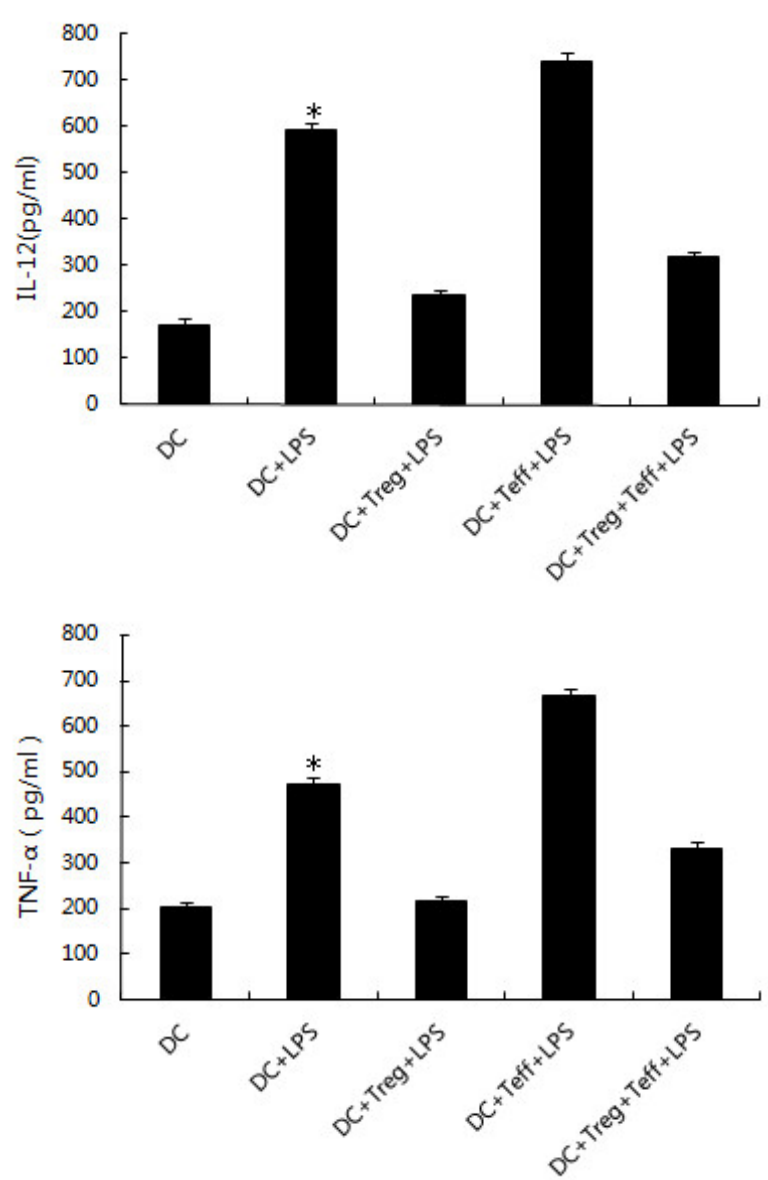

Figure 4. HCC-derived Tregs can Effectively Inhibit the Secretion of Proinflammatory Cytokines by Splenic DCs. Splenic DCs were cultured in medium alone, or with Tregs or Teff cells or a mix of both cells at a 1:1 ratio, and stimulated by LPS. HCC-derived Tregs obviously suppress secretion of IL-12 and TNF- $\alpha$ by DCs, even in the Treg, DC and Teff mixed group, while Teff cells enhance the secretion of IL-12 and TNF- $\alpha$ by DCs. Results are mean \pm sd of triplicate wells. *indicates significant difference $(p<0.05)$ when compared to other four groups 
produced even in the presence of various effector cells and contribute to in vivo Treg-mediated suppression.

Tumor-derived Tregs inhibit IL-12 and TNF- $\alpha$ secretion by DCs even in the presence of Teff cells

Activated DCs can secrete high levels of IL-12 and TNF- $\alpha$ which are important for the stimulation of $\mathrm{T}$ cells. To further address the role of tumor-derived Tregs on splenic DCs activation, cytokines were measured by ELISA in the supernatants of the cocultures described above. The level of IL-12 and TNF- $\alpha$ in the supernatant of DCs stimulated by LPS was detected higher than that in DCs cocultured with Tregs or a mix of Tregs and Teff cells. IL-12 and TNF- $\alpha$ production could be effectively inhibited by Tregs, even in the presence of Teff cells. In contrast, the production of IL-12 and TNF- $\alpha$ by splenic DCs was increased by the presence of Teff cells (Figure 4).

\section{Discussion}

Tregs play an important role in multiple immunoregulatory process including prevention of autoimmune diseases by maintaining self-tolerance, suppression of allergy and pathogen-induced immunopathology, regulation of the effector class of the immune response, etc (Kohl, 2007; Corthay, 2009; Fu et al., 2011). The relationship between Treg and cancer has been studied for a long time and accumulating researches have shown that Tregs are involved in tumor immunity. In our experiments, we found an increase of Treg/CD4+ in peripheral blood of HCC mice. The increased number of Tregs may participate in tumor development or affect the course of the disease.

Previous studies have concentrated on the interaction between Treg and bone marrow-derived DC. Although conventional Tregs have been shown to inhibit T cells and APCs (Misra et al., 2004; Onishi et al., 2008), we do not know the HCC-derived Tregs also have same inhibition on splenic DCs. To further understand the antitumor immuntiy of Tregs invloved in hepatocellular carcinoma, we researched on the mechanism between HCC-derived Treg and splenic DC. DCs exist in two functionally and phentypically distinct states, immature and mature. Immature DCs express low levels of MHC classIand II, and costimulatory molecules such as CD80/86. After detecting microbial products or proinflammatory cytokines, immature DCs transform into mature DCs and the MHC classI, costimulatory molecules and T cell adhesion molecules are all upregulated (Mellman et al., 2001). Mature DCs can also synthesize high levels of IL12 which enhances both innate and acquired immunity. LPS is one of the most potent activators of DCs (Auray et al., 2010). Most DCs in peripheral tissues in situ are of the immature phenotype including splenic DCs which consitutes 1-1.5\% spleen cells (Bonifaz et al., 2002). Teff cells control adaptive immunity against pathogens and cancer by activating other effector immune cells. Our experiments demonstrate that isolated splenic DCs express low levels of CD80/86. DCs co-cultured with Teff cells lead to an up-regulation in costimulatory molecule CD80/86 while DCs co-cultured with HCC-derived Tregs lead to a lower expression of CD80/86. This CD80/86 down-regulation occur even in the mixed culture of Tregs, Teff cells and DCs. Tregs keep low level of CD80/86 on DCs, so that DCs can not effectively activate and prolifer Teff cells. Tregs derived from HCC mice can also suppress the secretion of proinflammatory cytokine IL-12 and TNF- $\alpha$. Maturation of DCs is crucial for the initiation of immunity, but Tregs interfere with the maturation of DCs through down-regulation costimulatory molecule on DCs and inhibition the production of proinflammatory cytokines by DCs to make them to be poor APCs. Defective function of DC is one of important mechanisms of tumor escape from immune system control.

In conclusion, the study provides the evidence for increased prevalence of Tregs in peripheral blood of mice with HCC and extends the knowledge on interaction of tumor-derived Tregs and splenic DCs. We show that tumorderived Tregs inhibit maturation and antigen-presenting function of splenic DCs through down-regulation CD80/ CD86 expression on DCs and inhibit cytokine secretion by DCs even in the presence of Teff cells. The findings may be helpful for tumor immunotherapy.

\section{References}

Ahmed Ali HA, Di J, Mei W, et al (2014). Antitumor activity of lentivirus-mediated interleukin-12 gene modified dendritic cells in human lung cancer in vitro. Asian Pac J Cancer Prev, 15, 611-6.

Auray G, Facci MR, van Kessel J, et al (2010). Differential activation and maturation of two porcine DC population follow TLR ligand stimulation. Mol Immunol, 47, 2103-11.

Bonifaz L, Bonnyay D, Mahnke K, et al (2002). Efficient targeting of protein antigen to the dendritic cell receptor DEC-205 in the steady state leads to antigen presentation on major histocompatibility complex class Iproducts and peripheral CD8+ T cell tolerance. J Exp Med, 196, 1627-38.

Cabrera R, Ararat M, Eksioglu EA, et al (2010). Influence of serum and soluble $\mathrm{CD} 25$ ( $\mathrm{SCD} 25)$ on regulatory and effector T cell function in hepatocellular carcinoma. Scand J Immunol, 72, 293-301.

Corthay A (2009). How do regulatory T cells work? Scand $J$ Immunol, 70, 326-36.

Forner A, Llovet JM, Bruix J (2012). Hepatocellular carcinoma. Lancet, 379, 1245-55.

Fu T, Zhang P, Feng L, et al (2011). Accelerated acute allograft rejection accompanied by enhanced T-cell proliferation and attenuated treg function in RBP-J deficient mice. $\mathrm{Mol}$ Immunol, 48, 751-9.

Herman S, Krenbek D, Klimas M, et al (2012). Regulatory T cells form stable and long-lasting cell cluster with myeloid dendritic cells (DC). Int Immunol, 24, 417-26.

Hu JL, Yang Z, Tang JR, et al (2013). Effects of gastric cancer cells on the differentiation of Treg cells. Asian Pac J Cancer Prev, 14, 4607-10

Hu YX, Li M, Jia XH, et al (2013). HPV16 CTLepitope peptideactivated dendritic cell and natural killer co-culture for therapy of cervical cancer in an animal model. Asian Pac J Cancer Prev, 14, 7335-8

Jemal A, Bray F, Center MM, et al (2011). Global cancer statistices. CA Cancer J Clin, 61, 69-90.

Kohl J, Wills-Karp M (2007). Complement regulates inhalation tolerance at the dendritic cell/T cell interface. Mol Immunol, 44, 44-56.

Liu Z, Kim JH, Falo LD Jr, et al (2009). Tumor regulatory T 
cells potently abrogate antitumor immunity. J Immunol, 182, 6160-7.

Onishi Y, Fehervari Z, Yamaguchi T, et al (2008). Foxp3+ natural regulatory $\mathrm{T}$ cells preferentially form aggregates on dendritic cells in vitro and actively inhibit their maturation. PNAS, 105, 10113-8.

Ramanathan P, Ganeshrajah S, Kamalalayam R, et al (2014). Development and clinical evaluation of dendritic cell vaccines for HPV related cervical cancer- a feasibility study. Asian Pac J Cancer Prev, 15, 5909-16

Shen Z, Zhou S, Wang Y, et al (2010). Higher intratumoral infiltrated Foxp3+ Treg numbers and Foxp3+/CD8+ ratio are associated with adverse prognosis in resectable gastric cancer. J Cancer Res Clin Oncol, 136, 1585-95.

Unitt E, Rushbrook SM, Marshall A, et al (2005). Compromised lymphocytes infiltrate hepatocellular carcinoma: the role of T-regulatory cells. Hepatol, 41, 722-30.

Wan WJ, Tao Z, Gu W, et al (2013). Variation of blood T lymphocyte subgroups in patients with non-small cell lung cancer. Asian Pac J Cancer Prev, 14, 4671-3.

Zhan HL, Gao X, Zhou XF, et al (2012). Presence of tumourinfiltrating foxp3+ lymphocytes correlates with immature tumour angiogenesis in renal cell carcinomas. Asian Pac J Cancer Prev, 13, 867-72. 